\title{
An investigation of unsteady 3-D effects on trailing edge flaps
}

\author{
Eva Jost, Annette Fischer, Galih Bangga, Thorsten Lutz, and Ewald Krämer \\ Institute of Aerodynamics and Gas Dynamics, University of Stuttgart, Pfaffenwaldring 21, \\ 70569 Stuttgart, Germany \\ Correspondence to: Eva Jost (e.jost@iag.uni-stuttgart.de)
}

Received: 13 December 2016 - Discussion started: 15 December 2016

Revised: 22 March 2017 - Accepted: 10 April 2017 - Published: 16 May 2017

\begin{abstract}
The present study investigates the impact of unsteady 3-D aerodynamic effects on a wind turbine blade with trailing edge flap by means of computational fluid dynamics (CFD). Harmonic oscillations are simulated on the DTU $10 \mathrm{MW}$ rotor with a morphing flap of $10 \%$ chord extent ranging from 70 to $80 \%$ blade radius. The deflection frequency is varied in the range between 1 and $6 \mathrm{p}$. To quantify 3-D effects, rotor simulations are compared to 2-D airfoil computations and the 2-D theory by Theodorsen. It was found that the deflection of the flap on the 3-D rotor causes a complex wake development and induction which influences the loads over large parts of the blade. In particular, the rotor near wake with its trailing and shed vortex structures revealed a great impact. Trailing vorticity, a 3-D phenomenon, is caused by the gradient of bound circulation along the blade span. Shed vorticity originates from the temporal bound circulation gradient and is thus also apparent in 2-D. Both lead to an amplitude reduction and shed vorticity additionally to a hysteresis of the lift response with regard to the deflection signal in the flap section. A greater amplitude reduction and a less pronounced hysteresis is observed on the 3-D rotor compared to the 2-D airfoil case. Blade sections neighboring the flap experience, however, an opposing impact and hence partly compensate for the negative effect of trailing vortices in the flap section with respect to integral loads. Comparisons to steady flap deflections at the 3-D rotor revealed the high influence of dynamic inflow effects.
\end{abstract}

\section{Introduction}

The reduction of ultimate and fatigue loads plays an important role in today's wind energy research. In the background of economic efficiency, load alleviation systems bare potential to reduce rotor weight and costs, to increase the turbine reliability or allow a further enlargement of the rotor radius and thus power output. One promising concept to reduce dynamic load fluctuations are trailing edge flaps applied to the outer part of the rotor blade. As flaps are able to increase or decrease the local lift by adapting the deflection angle, it is possible to partly compensate for load variations due to variations of the effective inflow angle and velocity.

Over the last years, several investigations showed the potential of the flap concept, such as a test on a full-scale turbine performed by Castaignet et al. (2014). In aero-elastic simulations, fatigue load reductions up to approximately
$30 \%$ have been found for a trailing edge flap covering up to $25 \%$ of the blade span of a $5 \mathrm{MW}$ turbine (Barlas et al., 2012a). In most of the numerical studies the aerodynamic loading was computed by blade element momentum (BEM) codes (e.g., Bernhammer et al., 2016; Chen et al., 2017; Ungurán and Kühn, 2016), which have been extended with different engineering models to account for the unsteady flow (e.g., Bergami and Gaunaa, 2012). As viscous and unsteady aerodynamics have a great influence on dynamically deflected flaps (Leishman, 1994), it is, however, important to also apply higher-fidelity models and gain knowledge of the flow physics. In this respect, a lot of studies have been performed on 2-D airfoils, for example by Troldborg (2005) and Wolff et al. (2014) using computational fluid dynamics (CFD). Comparisons of simulation methods with different aerodynamic fidelities were performed by Bergami et al. 
(2015) in 2-D. For the 3-D wind turbine rotor, only few publications based on higher-fidelity aerodynamic models are available. In 2012 Barlas et al. (2012b) compared CFD to BEM predictions for a rotor with trailing edge flap in an artificial half-wake scenario and found a reasonably good agreement with regard to the complexity of the test case. Leble et al. (2015) investigated trailing edge flaps on the 3D rotor as part of the European AVATAR project and proved the load alleviation potential using a CFD approach. Several comparisons of codes with different aerodynamic fidelities can also be found in the AVATAR project reports (Manolesos and Prospathopoulus, 2015; Ferreira et al., 2015; Aparicio et al., 2016b). A benchmarking within the European INNWIND.EU project (Jost et al., 2015a; Barlas et al., 2016) showed, however, that there are still differences between the results of CFD simulations and BEM methods which need to be analyzed. While a previous investigation focused on the analysis of static flap deflection angles (Jost et al., 2016) by means of CFD, the main objective of the present work is to study the influence of unsteady 3-D effects on the example of harmonically oscillating morphing flaps.

Different deflection frequencies ranging from 1 to $6 p$ are analyzed on the DTU $10 \mathrm{MW}$ rotor (Bak et al., 2013) at rated operational conditions. These frequencies are considered a realistic operational range for active load alleviation. The investigated flap layout consists of a single morphing flap ranging from 70 to $80 \%$ blade radius with $10 \%$ local chord extent. This limited dimension along the blade span was chosen to obtain a high impact of 3-D effects. In all cases the flap oscillates with an amplitude of $10^{\circ}$. It should be noted that the present work does not aim towards an assessment of the flap concept. The objective is to investigate unsteady 3$\mathrm{D}$ aerodynamic effects caused by trailing edge flaps and to obtain deeper knowledge about the dominant phenomena as fundamental basis for an enhancement of engineering tools commonly used for load calculations. In this respect aeroelasticity is not considered since, on a flexible blade, pitching and plunging movements are superimposed onto the flap oscillation and a distinction of the isolated effects would be difficult.

\section{Aerodynamic effects of trailing edge flaps}

\subsection{Two-dimensional airfoil}

Trailing edge flaps are able to increase or decrease the airfoil lift for respectively positive (downwards) or negative (upwards) deflections due the change in the airfoil camber. As exemplarily displayed in Fig. 1, this leads to a vertical shift of the lift coefficient $c_{1}$ over angle of attack (AoA) $\alpha$ curve. This possible lift increase is, however, mostly connected to an increase in drag as depicted in the drag coefficient's $c_{\mathrm{d}}-\alpha$ plot. Additionally, the moment coefficient around the quarter chord point $c_{\mathrm{m}}$ is also significantly influenced by the change in the airfoil shape. In general the flap concept aims towards
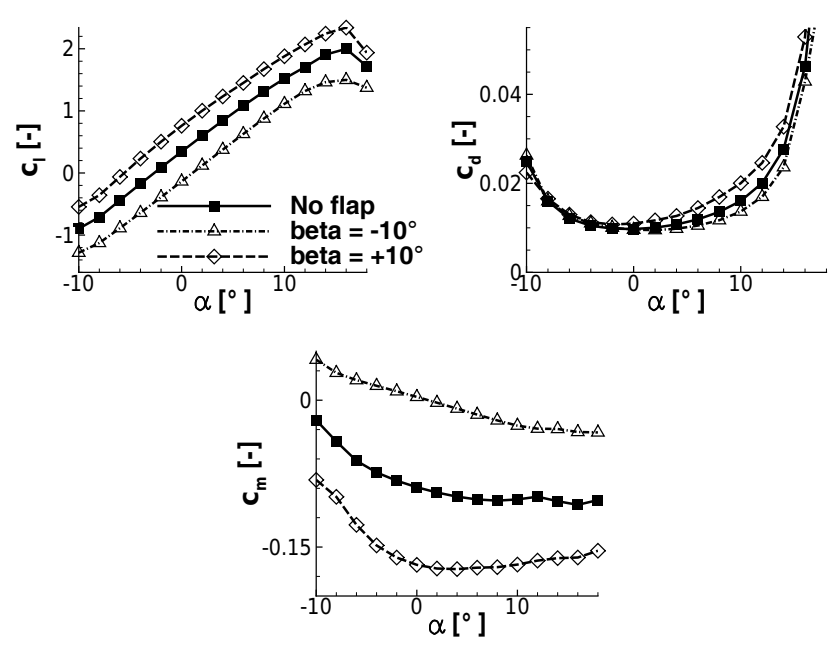

Figure 1. Example of flap deflection on $c_{1}, c_{\mathrm{d}}$ and $c_{\mathrm{m}}$ (FFAw3-241 airfoil, Reynolds number $R e=15.57 \times 10^{6}$, Mach number $M=0.2)$.

reducing the overall load fluctuation, but in particular the dominant out-of-plane forces and blade root bending moment. They are primarily influenced by the lift coefficient.

\subsection{Three-dimensional rotor blade}

The increase or decrease in lift in a blade section with trailing edge flap influences the aerodynamic phenomena in most parts of the rotor blade. A qualitative illustration of the vortex development around a rotor blade with deflected flap can be given on the basis of potential flow theory as illustrated in Fig. 2. It shows the vortex system with positive flap deflection in spatial (panel a) and temporal (panel b) consideration.

Due to the spatial gradient of bound circulation along the blade radius, a vortex sheet trails the rotor blade. In the flap section the bound circulation increases locally due to the change in camber. This leads to higher gradients at the flap edges and hence stronger trailing vortices at these locations. Outboard at the blade tip, the tip vortex is shown. Wake vorticity caused by radially changing bound circulation is commonly referred to as trailed vorticity.

Generally, the efficiency of the flap with regard to local lift increase or decrease is reduced by trailed vorticity in the 3-D case. The flap deflection causes an additional downwash or upwash in the flap section. This leads to a respectively lower or higher effective AoA in the 3-D case and consequently to induced drag in relation to the baseline AoA. It is worth noting that, with respect to the case without flap deflection $\left(\beta=0^{\circ}\right)$, the induced drag is increased in the case of positive deflections and decreased in the case of negative deflections. The adverse effect of trailing vortices in the flap section is, however, countered by a positive effect in the blade parts adjacent to the flap section. Caused by the sign change in induced velocities over the flap edge, the described effects 
(a)

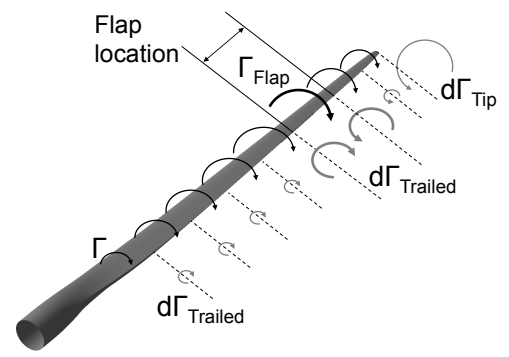

(b)

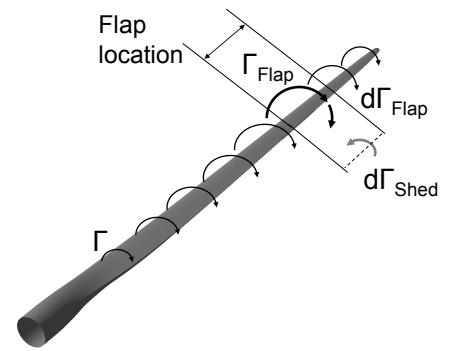

Figure 2. Sketch of the bound circulation along a wind turbine blade with trailing (a) and shed (b) vorticity (part a: Jost et al., 2017).

for the flap section are experienced in the opposite way at these blade parts. With regard to integral loads such as power and thrust, this effect opposes the negative impact of trailing vortices in the flap section.

The temporal consideration (Fig. 2b) displays an increase in bound circulation caused by an increase in the flap angle. This causes shed vorticity with opposed sense of rotation. Shed vortex structures re-induce velocities at the blade location and lead to a change in the effective AoA which in turn influences blade loads. Wake vorticity linked to temporal changes in bound circulation is called shed vorticity.

\subsection{Theodorsen theory}

Shed vorticity has been analyzed by Theodorsen and Garrick (1942) as well as by Leishman (1994) for the 2-D case of an airfoil with flap. Theodorsen and Garrick derived an analytical solution for the unsteady airfoil response caused by sinusoidal flap actuation based on his theory from 1935 for thin airfoils (Theodorsen, 1935). This solution is dependent on the reduced frequency (Eq. 1), one of the most important characteristic parameters when it comes to unsteady aerodynamics (Leishman, 2002). It is a measure of the unsteadiness of a problem as relation between frequency $f$ and chord length $c$ to the inflow velocity $v_{\text {inf }}$.

$k=\frac{\pi \cdot f \cdot c}{v_{\text {inf }}}$

According to Theodorsen's method the lift is given by

$$
\begin{aligned}
c_{1}(t) & =\underbrace{2 \pi C(k)\left(\frac{F_{10} \beta}{\pi}+\frac{F_{11} \dot{\beta} c}{4 \pi v_{\text {inf }}}\right)}_{1} . \\
& +\underbrace{\frac{c}{2 v_{\mathrm{inf}}^{2}}\left(-v_{\mathrm{inf}} F_{4} \dot{\beta}-\frac{c}{2} F_{1} \ddot{\beta}\right)}_{2} .
\end{aligned}
$$

The derived function consists of two terms: a first term which represents the circulatory forces connected to the bound circulation, and a second term which accounts for added mass effects. This second term is mostly referred to
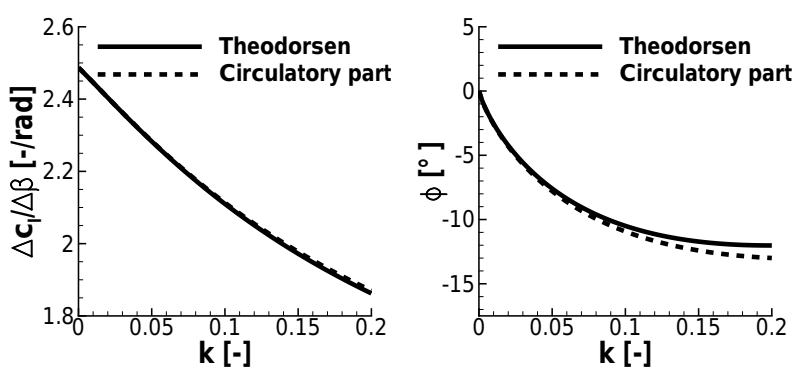

Figure 3. Lift amplitude in relation to flap amplitude $\Delta c_{1} / \Delta \beta$ and phase shift according to Theodorsen.

as non-circulatory part and depicts the influences of the inertia of the fluid. In this function, $\beta$ represents the instantaneous flap angle and its time derivatives. The coefficients $F_{1}$ to $F_{11}$ are geometric terms solely dependent on the relative flap length to chord. For their definition it shall be referred to Leishman (1994). The function $C(k)$ is the complex Theodorsen function which accounts for the effects of the shed wake.

The instantaneous lift coefficient $c_{1}(t)$ can be analyzed with regard to the amplitude $\Delta c_{1}$ and phase shift $\phi$ of the lift response with respect to the input flap signal. $\Delta c_{1}$ can also be evaluated in relation to the amplitude of flap deflection $\left(\Delta c_{1} / \Delta \beta\right)$. Figure 3 shows the solution for a flap length of $10 \%$ chord as function of the reduced frequency $k$. In the diagram two curves are plotted: a solid curve which represents the solution of the complete function $c_{1}(t)$, and a dashed curve which shows the solution if only the circulatory components are regarded and apparent mass effects are neglected. No major difference between both curves is apparent in $\Delta c_{1} / \Delta \beta$. It is continuously decreasing with $k$ in the displayed range which is applicable for this work as the investigated flap frequencies correspond to reduced frequencies of $k=0.024-0.147$ at mid-flap position. An increasing lag can be observed in phase shift, which is more pronounced if only circulatory components are included. While below a value of roughly 0.1 the differences between both curves are small, higher discrepancies are observed at larger $k$ when apparent mass effects become increasingly dominant. In conclusion it is, however, found that the non-circulatory contri- 
bution is in the investigated range of reduced frequency of minor importance.

Additionally to the lift equation, Theodorsen developed functions for $c_{\mathrm{m}}$, the pressure drag $c_{\mathrm{dp}}$ and the flap hinge coefficient $c_{\mathrm{m}}$,fh. The reader is referred to Hariharan and Leishman (1996) for their definition. Theodorsen's derivations include the assumptions of thin airfoils and 2-D flow. Both are not applicable for the aerodynamics of a modern wind turbine since current developments in blade design tend towards thicker airfoils for increased stiffness. But since the theory is well known and commonly used to determine unsteady aerodynamic characteristics, it is compared to the obtained results.

\section{Numerical approach}

\subsection{Simulation process chain}

The process chain for the simulation of wind turbines, which was developed at the Institute of Aerodynamics and Gas Dynamics (IAG), University of Stuttgart (Schulz et al., 2016), is used in the present work. The main part constitutes the CFD code FLOWer of the German Aerospace Center (DLR) (Kroll and Fassbender, 2002).

FLOWer is a compressible code that solves the 3-D Reynolds-averaged Navier-Stokes (RANS) equations in integral form. The finite-volume numerical scheme is formulated for block-structured grids. A second-order central discretization with artificial damping is used to determine the convective fluxes, which is also called the JamesonSchmidt-Turkel (JST) method. Transient simulations make use of the implicit dual-time-stepping scheme. To close the RANS equation system, several state-of-the-art turbulence models can be applied, such as the SST model by Menter (1994) used in this study. FLOWer offers the use of the CHIMERA technique for overlapping meshes which is applied in the simulation of 3-D wind turbines.

Grid generation is widely automated with scripts for 2$\mathrm{D}$ airfoils and 3-D rotor blades. The generation of the blade grid, for example, is conducted with Automesh, a script developed at the IAG for the commercial grid generator Gridgen by Pointwise. The blade grids are of $\mathrm{C}$ type with a tip block and coning towards the blade root in order to connect to the turbine spinner. Spinner and nacelle are typically included in the simulations. In the case of pure rotor simulations as performed in this study, the computational domain is modeled as a $120^{\circ}$ model with periodic boundary conditions on each side to reduce computing efforts. For the present study, this means that the flaps of all blades are deflected simultaneously.

On the post-processing side, again several scripts are available for the analysis of the simulations. Loads are calculated by the integration of pressure and friction distribution over the blade surface. Sectional distributions along the blade ra-
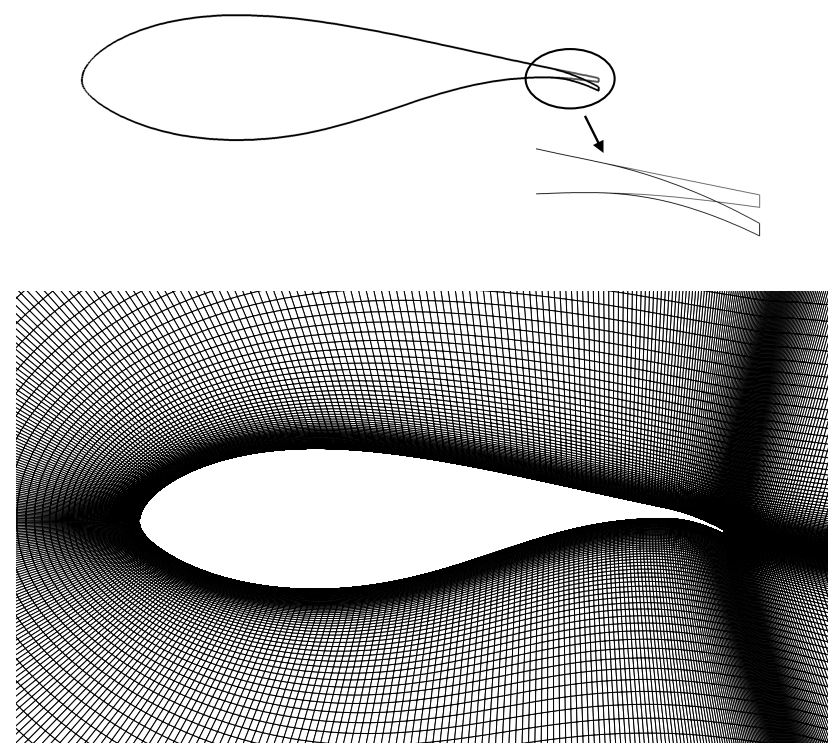

Figure 4. Methodology for 2-D deflection (Jost et al., 2016).

dius are determined similarly by dividing the blade into different radial sections.

\subsection{Trailing edge flap model}

Trailing edge flaps are modeled based on grid deformation in FLOWer. Therefore, the deformation module (Schuff et al., 2014) was extended by a polynomial function (Daynes and Weaver, 2012; Madsen et al., 2010) to describe the shape of the deflected flap.

$w=\varphi(x) \cdot \beta \quad \varphi(x)= \begin{cases}0 & 0 \leq x<(c-b) \\ \frac{(c-x-b)^{n}}{b^{n-1}} & (c-b) \leq x \leq c\end{cases}$

In Eq. (3) $c$ represents the chord length and $b$ the flap length. The result $w$ is the vertical change in the $y$ direction, while the movement in the $x$ direction is neglected for small deflection angles up to $10^{\circ}$. Using this function requires the chord to be aligned with the $x$ axis. The polynomial order $n$ is set to 2 for this investigation. In Fig. 4 the deformation methodology shown for a 2-D airfoil section. The undeformed and deformed airfoil surface are shown serving as input to the grid deformation algorithm, which computes the new simulation grid at each time step.

The approach for the blade mesh is displayed in Fig. 5. There is no separate grid for the flap part. It is integrated into the blade grid. The connection between the moving flap part and the remaining rigid blade surface is computed by the deformation algorithm which generates a smooth transition. At the location of these transitions the blade grid is radially refined to capture gradients in the flow field which are expected to occur. 


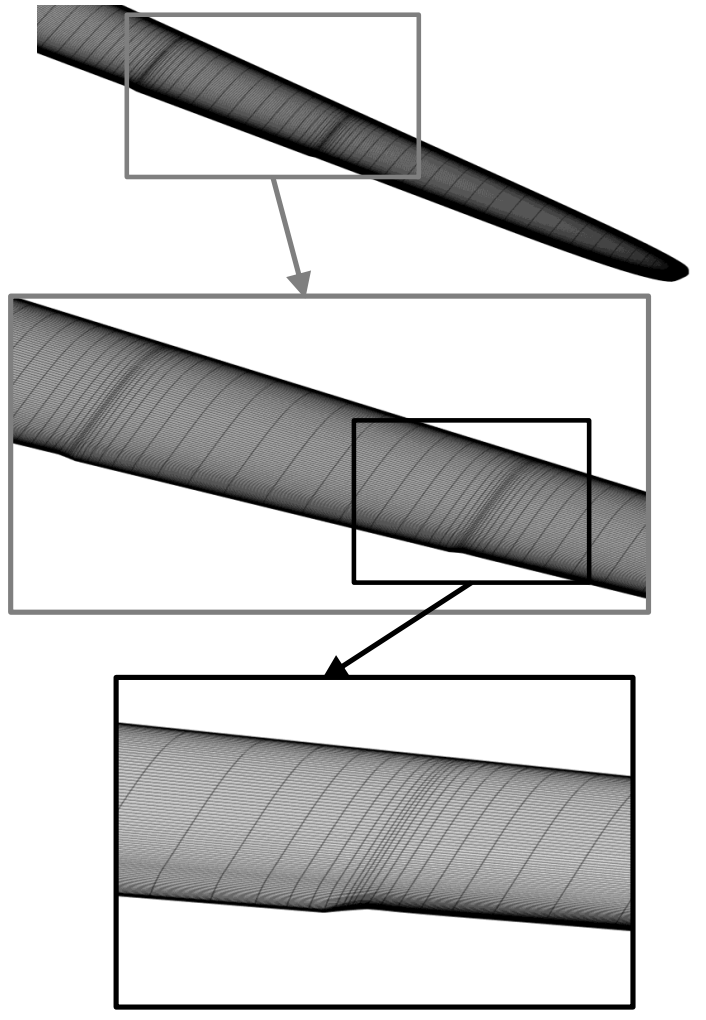

Figure 5. Methodology for 3-D deflection.

\subsection{Code-to-code validation of the simulations}

A baseline simulation setup for the DTU $10 \mathrm{MW}$ turbine without flap has been examined and validated by code-tocode comparison within the AVATAR project. For this purpose a simulation of the power curve on the basis of the stiff straight blade without precone was conducted in steady mode. The comparisons between the different codes of the project partners are presented by Sørensen et al. (2015). The FLOWer results showed good agreement with results obtained with EllipSys3D by DTU and MapFLow by NTUA. A detailed analysis of the FLOWer results with a special focus on the comparison of steady and unsteady simulations is performed in Jost et al. (2015b). A comparison of the simulations with flaps to the AVATAR project partners can be found in Ferreira et al. (2015) and Aparicio et al. (2016b).

\subsection{Grid generation}

For the 2-D airfoil simulations, the $75 \%$ blade cut of the DTU 10 MW turbine (FFA-w3-241 airfoil), which is the midflap position in the chosen trailing edge flap configuration, was extracted from the geometry. The airfoil grid was generated using a script for the commercial grid generator Pointwise. Approximately 180000 grid cells have been used with 417 surface nodes and 205 nodes in the wall-normal direc-
Table 1. Grid cells in the $120^{\circ}$ model.

\begin{tabular}{rrrrr}
\hline Blade & Spinner & Nacelle & Background & Total \\
\hline $8.16 \times 10^{6}$ & $1.39 \times 10^{6}$ & $1.45 \times 10^{6}$ & $10.65 \times 10^{6}$ & $21.65 \times 10^{6}$ \\
\hline
\end{tabular}

tion. Boundary layer resolution was chosen for $y_{\max }^{+} \sim 1$ and the farfield boundary is located at a distance of 150 chords.

For the rotor simulations, the setup used in the code-tocode validation was modified in order to simulate the rotor with trailing edge flap. Flap edge refinements were included into the blade grid and a higher resolved background grid was chosen to accurately capture wake effects. A separate grid convergence study of the blade grid was performed with a steady flap deflection $\pm 10^{\circ}$ and the results are presented in Jost et al. (2015a). The final setup used in the present study amounts to approximately 21.65 million cells. The distribution between the different grids is shown in Table 1 .

\subsection{Temporal discretization of the simulation setup with flaps}

Another critical issue with regard to the unsteady simulations is the temporal resolution meaning the choice of time step. Unsteady simulations in FLOWer make use of the dual-timestepping method as an implicit scheme. In this approach a pseudo-time is introduced into the equation system at each time step for which a steady solution is obtained. The method allows the choice of significantly larger time steps than those dictated by the Courant-Friedrichs-Lewy condition in explicit schemes. However, the actual eligible size is problem dependent. In most cases the time step is a trade-off between simulation accuracy and computational time and it is necessary to determine the largest possible time step that can still resolve the unsteady flow effects sufficiently. To analyze the influence of the temporal discretization within this study, a sensitivity study has been performed based on 2-D and 3-D simulations

First, the 2-D airfoil case (FFA-w3-241, $75 \%$ blade radius) is presented. The simulations have been performed at a realistic inflow extracted from the 3-D rotor case at rated operational conditions. These conditions are specified in Table 2 . At $75 \%$ radius, the Reynolds number was determined to 15.4 millions, Mach number to 0.2 and the AoA to $6.5^{\circ}$. For the determination of the time step influence, the high flap frequency corresponding to 6 times the rotational frequency at rated operational point $(6 p)$ was chosen, which corresponds to a reduced frequency of 0.147 . Results for $c_{1}$ and $c_{\mathrm{d}}$ are shown in Fig. 6. As similar findings were made for $c_{\mathrm{m}}$, it is not separately shown here but will be regarded in 3-D. Four different time steps have been selected for the study and 100 inner iterations are performed in the dualtime-stepping scheme for all investigated time steps except the small step of $0.028^{\circ}$, for which 30 inner iterations are regarded sufficient. As the results are transferred to the 3-D 


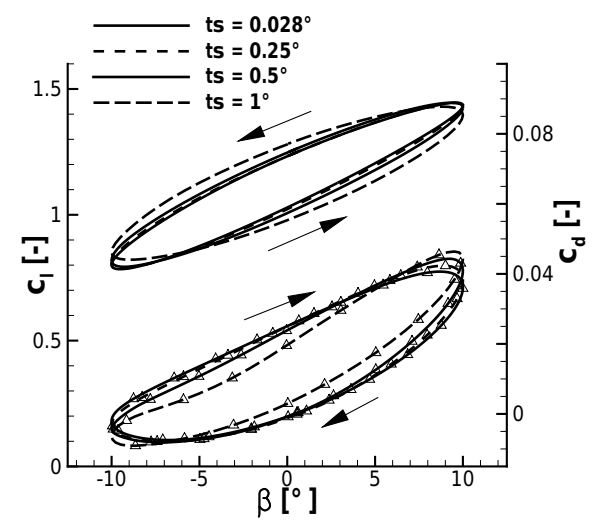

Figure 6. Influence of time step in 2-D, lift (no symbol) and drag $($ symbol $\triangle$ ).

Table 2. DTU $10 \mathrm{MW}$ turbine, rated operational conditions.

\begin{tabular}{rrrr}
\hline Wind speed & Rotational speed & Blade pitch & Tip speed ratio \\
\hline $11.4 \mathrm{~m} \mathrm{~s}^{-1}$ & $9.6 \mathrm{rpm}$ & $0^{\circ}$ & 7.86 \\
\hline
\end{tabular}

case later on, the different time steps are designated by the corresponding azimuth step in a rotor simulation at rated rotational speed. A time step of $0.028^{\circ}$ is for example equivalent to $4.8 \times 10^{-4} \mathrm{~s}$. This very small step correlates with 100 steps per convective time unit, which is in this case the chord length. With regard to the computational effort this time step is not realizable for the 3-D case, but serves as a reference in this 2-D study. All other discretizations are applicable for pure rotor simulations. In both aerodynamic coefficients the influence of the time step size is apparent, but the effect on drag is slightly more distinct. In general, lift and drag agree well for all resolutions except for $1^{\circ}$, where major differences are observed.

Another parameter of influence is the amount of inner iterations in the dual-time-stepping scheme, which is analyzed for the time step of $0.5^{\circ}$ with three different amounts of inner iterations, 50, 100 and 200. The results are displayed in Fig. 7. Again the results for the small time step of $0.028^{\circ}$ is shown as reference. Generally, it is observed that the temporal accuracy is more dependent on the total number of iterations per convective unit than the choice of time step or inner iterations. By comparing the plots in Figs. 6 and 7, it can be seen that, for example, the hysteresis for $1^{\circ}$ and 100 inner iterations is very similar to the curve with $0.5^{\circ}$ and 50 inner iterations. This conclusion can, however, not be transferred to separated flows, for which a small time step is needed to resolve effects correctly.

Based on these outcomes, simulations of the rotor model have been conducted in order to get an impression of the 3-D case. The flap is again oscillating with $6 \mathrm{p}$ frequency at rated operational condition. Similar time step sizes to those in the 2 -D case have been chosen, replacing $0.028^{\circ}$ with $0.125^{\circ}$ as

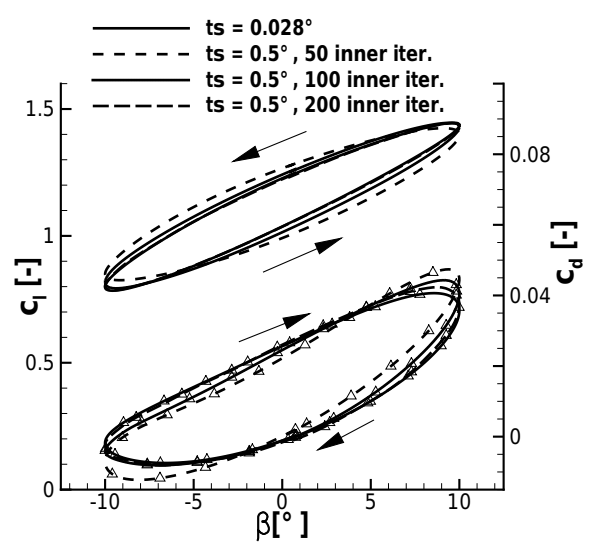

Figure 7. Influence of inner iterations in 2-D, lift (no symbol) and drag (symbol $\triangle$ ).

further halving. A total of 100 inner iterations are used. Figure 8 shows the resulting driving force, thrust and torsion moment variations at mid-flap position. The torsion moment is evaluated relative to the blade pitch axis and positive reducing the blade pitch. The forces and moment are normalized with the total mean value to allow an easier assessment of the differences. While thrust and torsion moment show good agreement for nearly all time step sizes, higher deviations are observed in the driving component in which the drag differences have a stronger impact. However, a convergence of the curve progressions with decreasing time step size can be observed, leading to small differences between 0.125 and $0.25^{\circ}$.

To conclude the temporal discretization study, a time step size of $0.25^{\circ}$ with 100 inner iterations for a flap frequency $6 \mathrm{p}$ shows sufficient accuracy in 3-D simulations as a trade-off to computational time. This corresponds to 240 steps per flap oscillation. In 2-D simulations smaller time steps correlated with the convective unit are feasible and consequently used.

\section{Results}

\subsection{Three-dimensional rotor simulations with oscillating flap}

First, results of the different flap frequencies are compared for the 3-D rotor simulations. All simulations have been conducted at rated conditions (see Table 2). As ambient conditions an air density of $1.225 \mathrm{~kg} \mathrm{~m}^{-3}$ and a temperature of $288.15 \mathrm{~K}$ are used. The simulations were started as a steadystate computation on two multi-grid levels with 8000 iterations each and a flap angle of $0^{\circ}$. This steady solution is then restarted in unsteady mode and simulated for the amount of revolutions required for converged loads. With regard to the Menter SST turbulence model, it should be mentioned that the required wall distances of each cell were computed only once at the simulation start and not updated in every time step. This was done since only a minor influence was found 
(a)

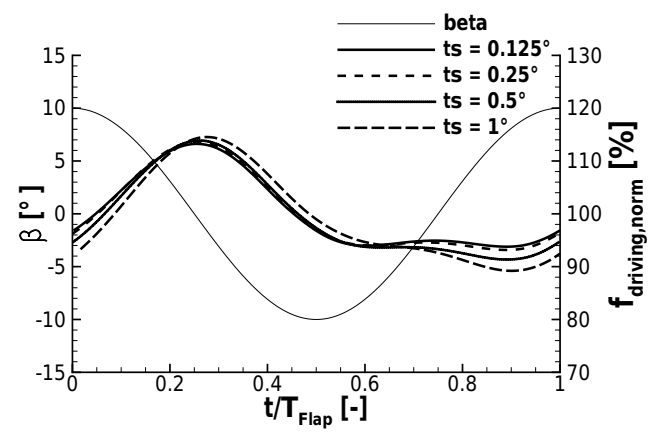

(b)

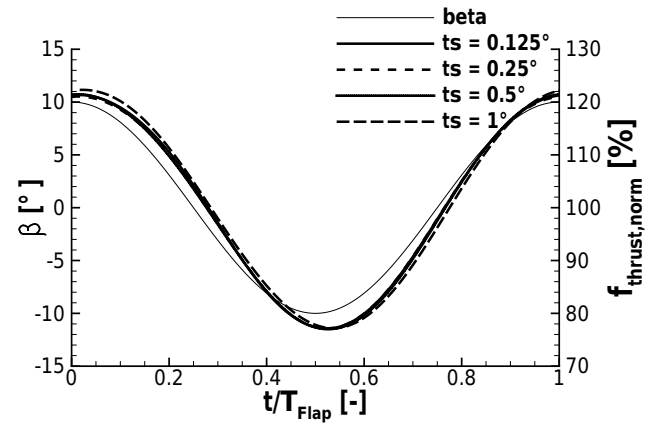

(c)

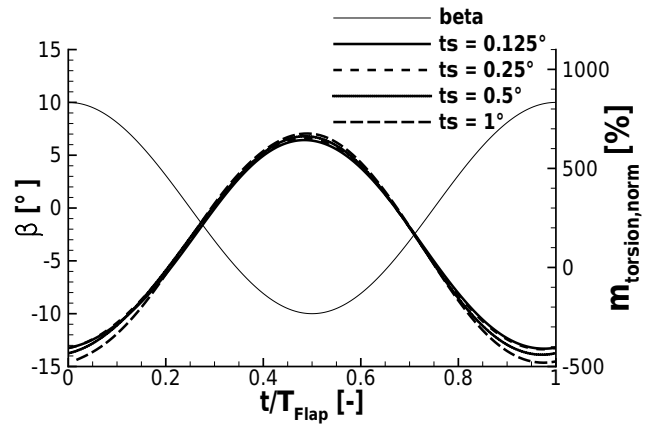

Figure 8. Influence of time step in 3-D, 6 p, $75 \%$ blade cut (driving force, $\mathbf{a}$; thrust, $\mathbf{b}$; and local torsion moment, $\mathbf{c}$ ).

in 2-D airfoil simulations and to save computation time as in 3-D the wall distance calculation is very time consuming. Please note that while in the previous time step study a cosine function is used as the deflection signal, now a sinus function is applied (Eq. 4).

$\beta(t)=10^{\circ} \cdot \sin \left(2 \pi t \frac{N}{T_{\text {Rotor }}}\right)$

$N=[1,2,3,6], T_{\text {Rotor }}=6.25 \mathrm{~s}$

The flap frequencies correspond to reduced frequencies of $k=0.024(1 \mathrm{p})-0.147(6 \mathrm{p})$ at mid-flap position. Figure 9 shows the results of integral power and thrust plotted over one rotor revolution. The effect of the flap can be seen clearly in both diagrams. Power and thrust are oscillating with the respective frequency. A higher frequency fluctuation is also apparent in the graphs, which results from unsteady flow separation at the cylindrical blade root. As illustrated in the (a)

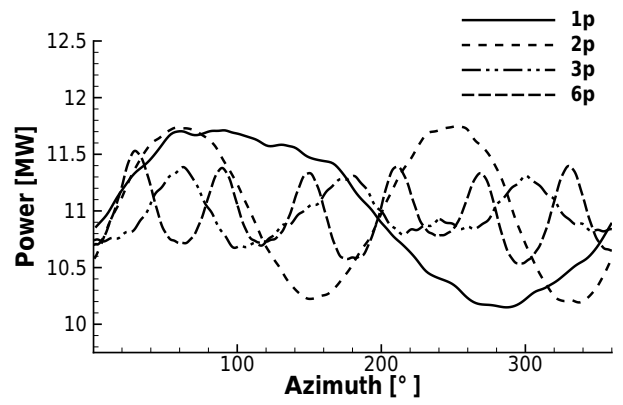

(b)

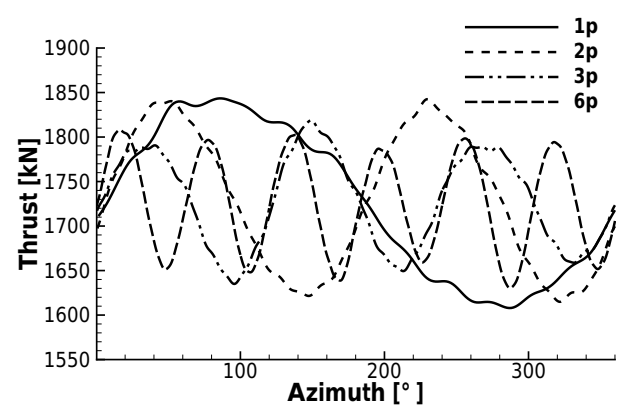

Figure 9. Integral rotor power (a) and thrust (b).

streamlines plot on the blade surface shown in Fig. 10, flow separation is dominant there. Due to this superposition of effects in the integral forces, it is necessary to regard sectional forces at a blade cut belonging to the flap part in order to investigate the flap effects.

In the following, like in the time step study, the $75 \%$ cut as mid-flap position was extracted from the simulations. Figure 11 shows the results of the local driving force, thrust and torsion moment at this location over one flap period for all simulated frequencies. Thrust shows the expected amplitude decrease and phase shifts with increasing frequency. A significant reduction of the amplitude is observed between the 2 and $3 p$ case, with 3 and $6 p$ showing a very similar curve progression. These phenomena will be analyzed in more detail in Sect. 4.4. Larger differences are observed in the driving component. For the 3 and $6 \mathrm{p}$ case, a second superimposed oscillation is visible from $t / T_{\text {Flap }} \approx 0-0.2$ and $t / T_{\text {Flap }} \approx 0.8-1$. This oscillation results from the overlay of lift and drag forces in the rotor plane. At higher frequencies drag shows a significant amplitude increase as seen in Fig. 6 and, additionally, $c_{1}$ and $c_{\mathrm{d}}$ are oscillating with different phases. The superposition of both force components leads to the curve progression seen in the 3 and $6 \mathrm{p}$ case. This phenomenon in driving force is especially present at operational conditions with a pitch angle of $0^{\circ}$, for which the impact of drag is high. Please note that in the plot of the local torsion moment (Fig. 11c), the $\beta$ axis is reversed in order to be able to better judge the phase shift. The moment shows an increase in amplitude with increasing flap frequency, mainly between the 2 and $3 p$ case like it was observed vice versa for thrust. The high frequencies 3 and $6 \mathrm{p}$ are again similar. All 


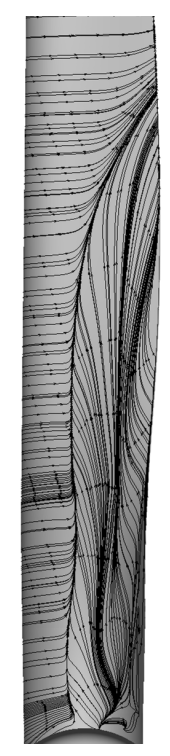

Figure 10. Blade root flow separation - suction side.

curves show a lead with respect to the flap signal. A more detailed elaboration of the torsion moment relative to the quarter chord point is also performed in Sect. 4.4.

In Figs. 12 and 13 sectional distributions of driving force, thrust and torsion moment over the blade radius are shown for the 1 and $6 \mathrm{p}$ case respectively. Four instantaneous solutions are plotted for maximum, minimum and $0^{\circ}$ flap deflection. Thrust shows the expected increase and decrease in the flap section with a smooth load distribution over the flap edges. This smoothing is a consequence of the positive effect of the flap deflection on neighboring blade sections as described in Sect. 2.2. While trailing vorticity reduces the effect of the flap in the flap section compared to 2-D, the sections next to the flap part produce higher/lower lift due to the induced upwash/downwash for respectively positive/negative flap angles. The change in sign in induced velocity caused by the flap edge vortices is also apparent in the driving force as significant steps are appearing at the transition between flap and rigid rotor part. An opposite behavior of sectional driving force in relation to thrust can be noticed by comparing the diagrams. When thrust increases locally in the flap area, the driving force decreases in relation to neighboring sections. This results again from the strong influence of drag on the driving component at rated wind turbine conditions and will be explained on the basis of the $1 \mathrm{p}$ case as follows. Due to the low reduced frequency in this case $(k=0.024)$, the influence of shed vorticity is still weak. For maximum positive deflection $\left(t / T_{\text {Flap }}=0.25\right)$ the increase in trailing vorticity causes a downwash in the flap section. This reduces the effective AoA and leads to a rise in induced drag in addition to the drag augmentation caused by the flap deflection itself. The overall drag increase is compensated for by the lift increase resulting from the flap deflection and relative to $0^{\circ}$ flap de- (a)

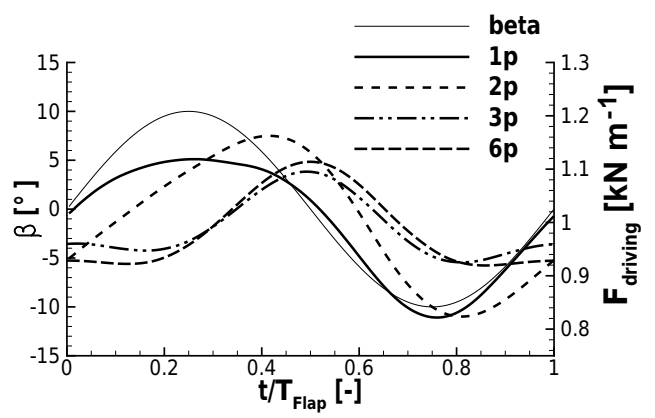

(b)

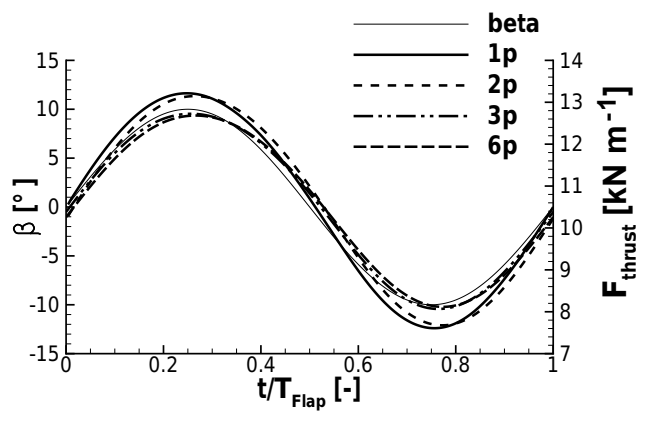

(c)

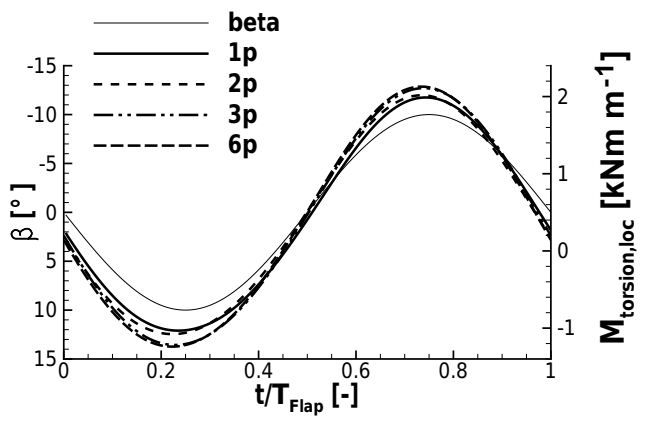

Figure 11. Variation in flap frequency, $75 \%$ blade cut (driving force, $\mathbf{a}$; thrust, $\mathbf{b}$; and local torsion moment, $\mathbf{c}$ ).

flection an increase in driving force is achieved. The neighboring sections to the flap experience an additional upwash in the case of positive deflections. Consequently, the induced drag reduces associated with the lift increase and these sections produce in total a higher sectional driving force. Similar observations are made vice versa for maximum negative deflections, but the driving force increase in the flap section is less pronounced compared to the decrease in the case of positive deflection. Further elaborations in this respect can be found in Sect. 4.3, in which lift and drag forces are extracted and compared. With regard to the torsion moment around the pitch axis, a strong oscillation is seen in the flap section with steep gradients at the flap edges. This torsion moment or $c_{\mathrm{m}}$ oscillation is typical for trailing edge flaps (Ferreira et al., 2015) and its effect on the overall performance of the flap concept needs to be investigated separately in an aero-elastic simulation when the blade is able to twist.

The differences caused by unsteady effects can also be observed in the plots. Larger variations of the forces are seen 
(a)

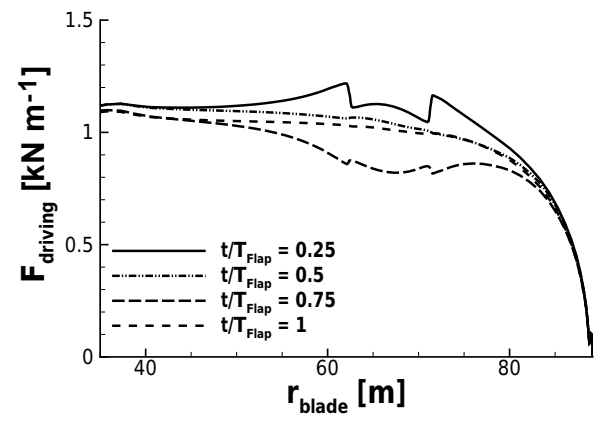

(b)

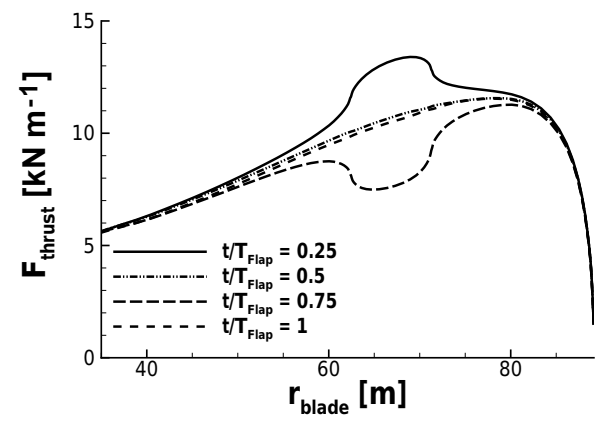

(c)

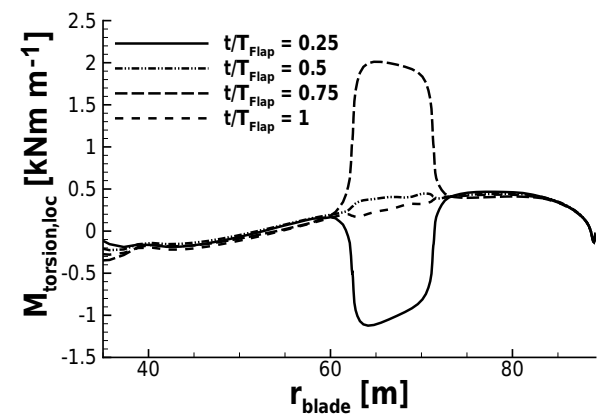

Figure $12.1 \mathrm{p}$ sectional forces (driving force, $\mathbf{a}$; thrust, $\mathbf{b}$; and local torsion moment, $\mathbf{c})$.

in the $1 \mathrm{p}$ case compared to the $6 \mathrm{p}$ case over the whole blade part influenced by the flap. In contrast the variation of the moment is slightly increasing. The hysteresis can especially be noticed at the time instance when the flap is positioned at $0^{\circ}$ deflection. While in the $1 \mathrm{p}$ case the sectional loads at increasing or decreasing flap angle are close together, in the $6 \mathrm{p}$ case larger differences are seen. For the decreasing flap angle at $t / T_{\text {Flap }}=0.5$ the loads are higher than for the increasing flap angle at $t / T_{\text {Flap }}=1$.

\subsection{Comparison to steady flap deflections}

In a first step to analyze the influence of unsteady effects in 3$\mathrm{D}$, the results are compared to the simulations of steady flap deflection for $\pm 10^{\circ}$. Like for the oscillating flap cases, the simulations were initiated in steady state with 16000 iterations and then restarted in unsteady mode for three turbine revolutions with a time step corresponding to $2^{\circ}$ azimuth. This approach is plausible because the flap area is charac- (a)

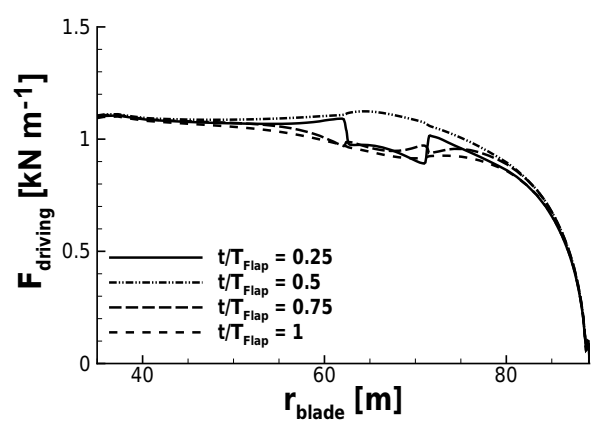

(b)

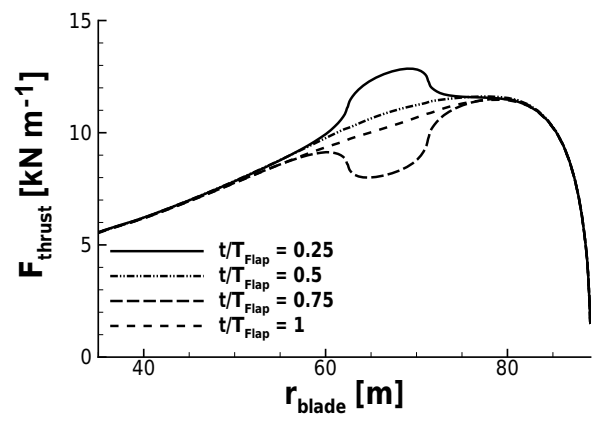

(c)

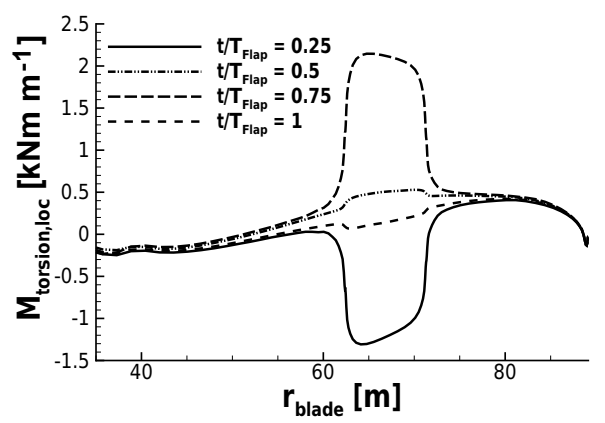

Figure 13. $6 \mathrm{p}$ sectional forces (driving force, $\mathbf{a}$; thrust, $\mathbf{b}$; and local torsion moment, $\mathbf{c})$.

terized by a steady flow situation as shown in similar studies (Aparicio et al., 2016b). Table 3 shows the mean integral loads of the third rotor revolution normalized with the respective value for $\beta=0^{\circ}$ for a relative comparison. By comparing to the results of the oscillating flap (Fig. 9), it can be noticed that in the oscillating cases an increase in power is possible, while for steady deflections this is not the case. A negative flap angle even leads to a higher power output than a positive deflection. This phenomenon is caused by the differences in axial induction. For positive deflections $c_{1}$ increases, more energy is extracted from the wind and consequently the axial inductions also rises. This leads to a lower AoA at the rotor blades, which reverses the effect of the flap with regard to power. The opposite is observed in the case of negative flap angles. But since less energy is extracted from the wind, a lower power output compared to the neutral flap case is still observed. In this background thrust also shows reasonable values. The magnitudes in the steady cases are lower compared to the $1 \mathrm{p}$ oscillating case, for which the ax- 
Table 3. Integral loads for steady deflection normalized with value for $\beta=0^{\circ}$.

\begin{tabular}{lrr}
\hline & $\beta=10^{\circ}$ & $\beta=-10^{\circ}$ \\
\hline Normalized power (\%) & 96.2 & 98.3 \\
Normalized thrust (\%) & 103 & 95.2 \\
\hline
\end{tabular}

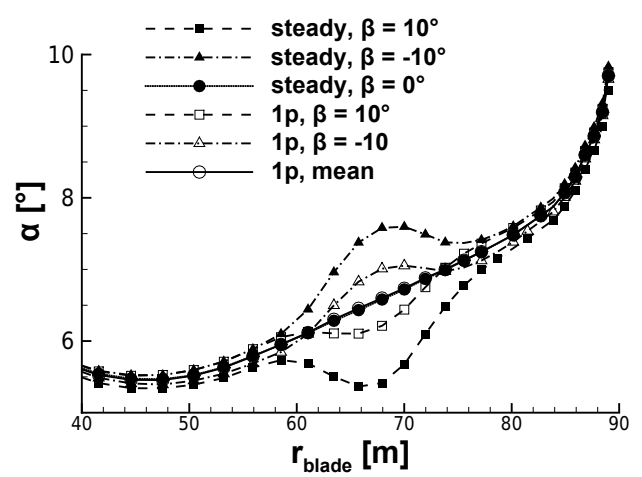

Figure 14. Extracted AoA according to Johansen and Sørensen (2004).

ial induction is not able to fully adjust to the changed load situation and consequently higher oscillations are possible.

To verify this hypothesis an extraction of the local AoA along the blade radius has been performed according to the reduced axial velocity method (Johansen and Sørensen, 2004). This method has proven to produce reasonable results (Bangga et al., 2016; Klein et al., 2014), but it is only applicable for steady inflow conditions. But since in the $1 \mathrm{p}$ case the reduced frequency is still very low with a value of 0.024 at mid-flap position, a quasi-steady approach is appropriate (Leishman, 2006). The method requires annular elements at different radii in front of and behind the rotor plane as input. These elements are placed at an axial distance of one local chord to the rotor blade for the present evaluation. The choice of this axial distance has, however, shown to have an influence on the results with maximum discrepancies of about $0.16^{\circ}$ when the axial distance is reduced for example to 0.2 local chords. Nevertheless, the results of the different cases can be compared to each other and give a qualitative and within this tolerance quantitative analysis. The plot displayed in Fig. 14 highlights the differences for steady and oscillating flap deflections and underlines that in unsteady cases the axial induction is only slowly adjusting. Consequently, dynamic inflow effects play an important role on trailing edge flaps, especially at lower flap frequencies.

Figures 15 and 16 show extracts from the flow field at an axial distance of one local chord in front of and behind the rotor blade for respectively maximum positive and flap negative angle. The contour of the axial velocity $u$ is displayed from a front view to the turbine, which means that the rotational direction is clockwise. The rotor blade is positioned

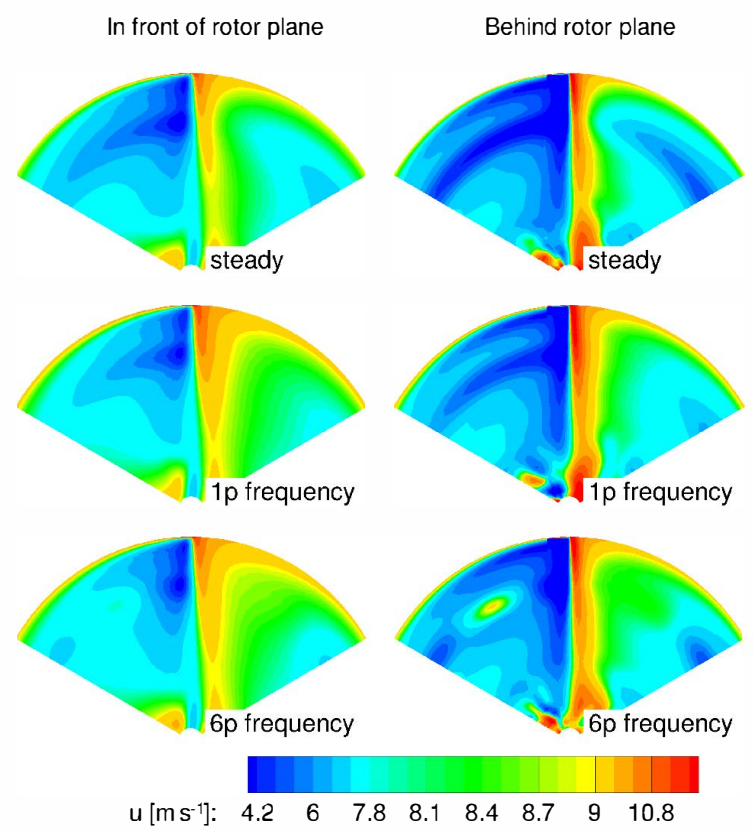

Figure 15. Flow field extracted at one local chord distance in front of and behind the rotor blade for $\beta=10^{\circ}$.

upright. In each figure three cases are shown: steady flap deflection, $1 \mathrm{p}$ oscillation and $6 \mathrm{p}$ oscillation. Clearly, the flow acceleration towards the blade and the reduction of the wind speed in the blade wake can be seen in all cases. In the flap section the opposing deflection can mainly be identified in the blade wake, where less reduction is observed for a negative angle and a higher reduction for a positive angle. By comparing steady deflections to the $1 \mathrm{p}$ frequency, the different axial induction can be seen. When comparing the 1 and $6 \mathrm{p}$ frequency, the different flap frequencies can also be noticed in the blade wake. In the $6 \mathrm{p}$ case at, for example, $\beta=10^{\circ}$, an area with increased velocity is apparent.

\subsection{Influence of varying AoA in 3-D}

As observed in the previous section, in the 3-D rotor case the local AoA is oscillating over a flap period as a result of dynamic inflow. This means from an aerodynamic point of view that two unsteady mechanisms are superimposed: pitch and flap oscillation. The objective of the present work is, however, to characterize and quantify unsteady 3-D effects solely due to flap deflection, and consequently some preliminary considerations have to be made. For this purpose the $1 \mathrm{p}$ frequency is regarded in the following, for which quasisteady assumptions are eligible and the reduced axial velocity method can be applied. The variation of the local inflow velocity and the AoA is shown in Fig. 17 for the mid-flap position. While the inflow velocity shows no major variations, the AoA oscillates with an amplitude of $0.6^{\circ}$. 


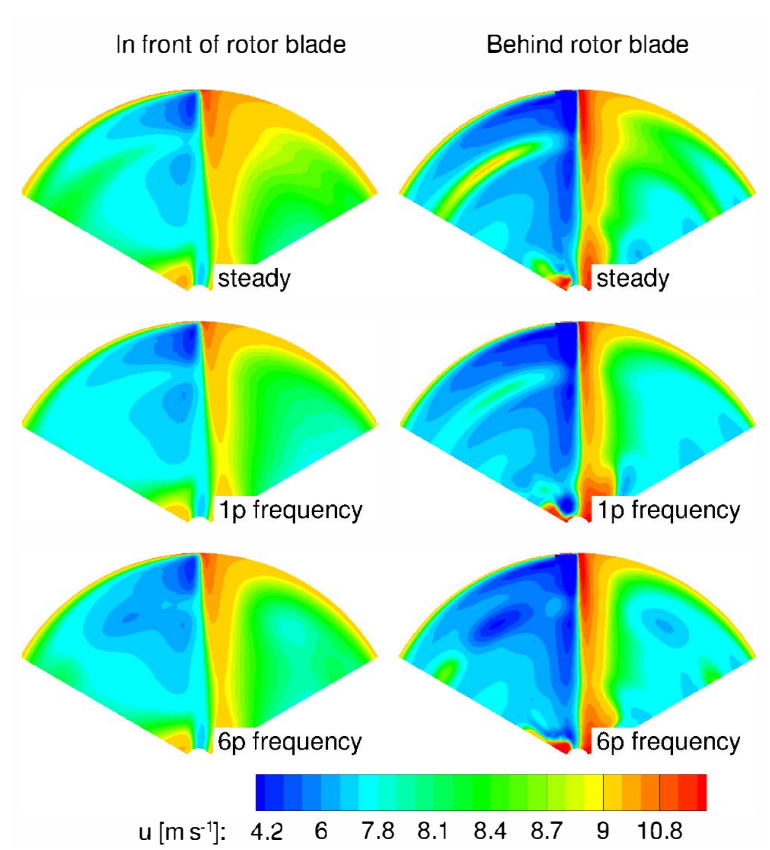

Figure 16. Flow field extracted at one local chord distance in front of and behind the rotor blade for $\beta=-10^{\circ}$.

When the instantaneous AoA is extracted from the 3-D simulation it includes the oscillations caused by both mechanisms, dynamic inflow and flap oscillation. The dynamic inflow oscillation represents an oscillation of the baseline AoA as a result of the variation of the axial induction of the turbine. In contrast the oscillations caused by the flap originate from the downwash of 3-D trailing vorticity, which changes the effective AoA. As the objective is to quantify 3-D trailing vorticity, the flap-caused AoA oscillation should be mimicked to the aerodynamic coefficients, while in theory the influence of the oscillation caused by dynamic inflow should be eliminated. A clear distinction between both oscillations is, however, not possible and requires further aerodynamic modeling. Nevertheless, the influence of the overall AoA oscillation on the 3-D extracted aerodynamic coefficients can be assessed.

Figure 18 presents the resulting $c_{1}$ and $c_{\mathrm{d}}$ variations in addition to the resulting variations for an averaged AoA of $6.5^{\circ}$ and local inflow velocity of $68 \mathrm{~m} \mathrm{~s}^{-1}$ ( $\left.c_{1 \text {, mean }}, c_{\mathrm{d} \text {,mean }}\right)$. The moment coefficient $c_{\mathrm{m}}$ is not dependent on the inflow direction, so that the evaluation of the AoA uncertainty for the $c_{\mathrm{m}}$ behavior can be excluded in this section. It can be seen that the AoA oscillations have only a minor influence on the value of $c_{1}$ but a strong impact on $c_{\mathrm{d}}$. This is reasonable as for the determination of $c_{1}$ and $c_{\mathrm{d}}$ in the 3-D case, the forces are integrated from the surface solution as driving force and thrust components at first and then transferred to the local inflow, also called the aerodynamic coordinate system. The procedure is shown in Fig. 19 for both components. To determine total lift and drag forces, both shares by driving force

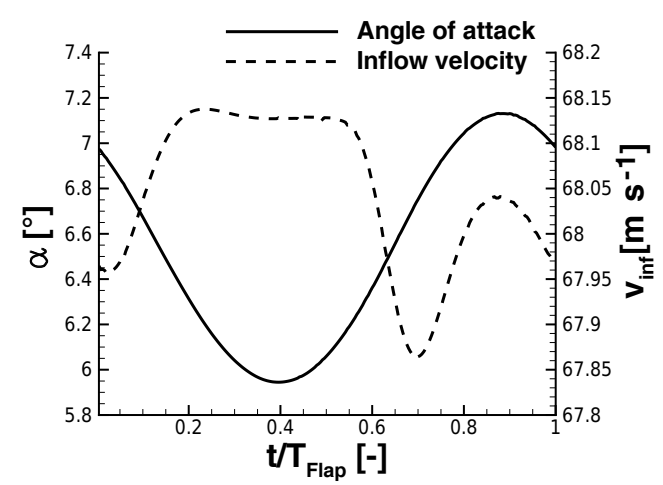

Figure 17. $1 \mathrm{p}$ instantaneous inflow conditions 3-D, $75 \%$ radius.

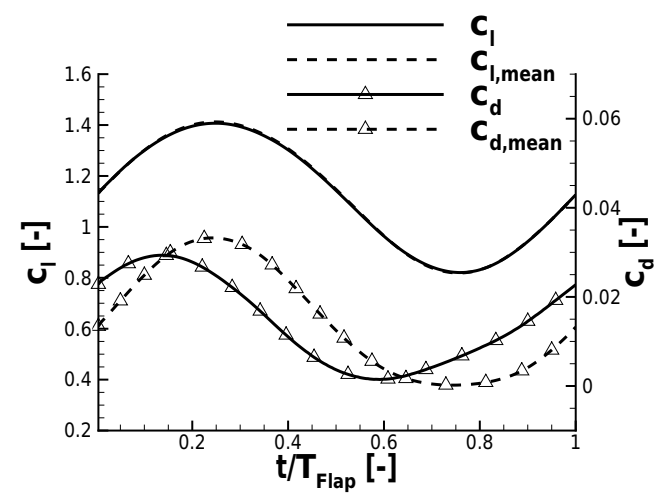

Figure 18. 1p comparison of lift and drag, 3-D instantaneous/3$\mathrm{D}$ mean, $75 \%$ radius.

and thrust are summed up. As the value of $c_{1}$ is roughly 100 times larger compared to $c_{\mathrm{d}}$, a projection difference of $0.6^{\circ}$ as observed in the $1 \mathrm{p}$ case has only a negligible impact on $c_{1}$. Consequently, the results for $c_{1}$ and $c_{1, \text { mean }}$ are very similar. However, $c_{\mathrm{d}}$ and $c_{\mathrm{d} \text {,mean }}$ differ strongly. Based on the previous considerations this difference consists of two parts: induced drag which originates from trailed vorticity and the drag resulting from the AoA oscillation caused by dynamic inflow.

The plot in Fig. 18 can be directly linked to the curves for driving force and thrust in the $1 \mathrm{p}$ case displayed Figs. 11 and 12. Thrust is dominated by $c_{1}$ and consequently the progressions over a flap period are very similar. The driving component is a superposition of both forces which are oscillating at a different phase. This can be noticed, for example, at the time instance when the flap is deployed to maximum deflection $\left(t / T_{\text {Flap }}=0.25\right) . c_{1}$ but also $c_{\text {d,mean }}$ is maximal and as a result the progression of driving force flattens. The phenomenon is observed in the opposite way at minimum deflection $\left(t / T_{\text {Flap }}=0.75\right)$.

With regard to the objective of this section, determining the influence of the AoA oscillation on the 3-D extracted aerodynamic coefficients, it can be concluded that the extracted $c_{1}$ is only marginally affected and it is appropriate 
(a)

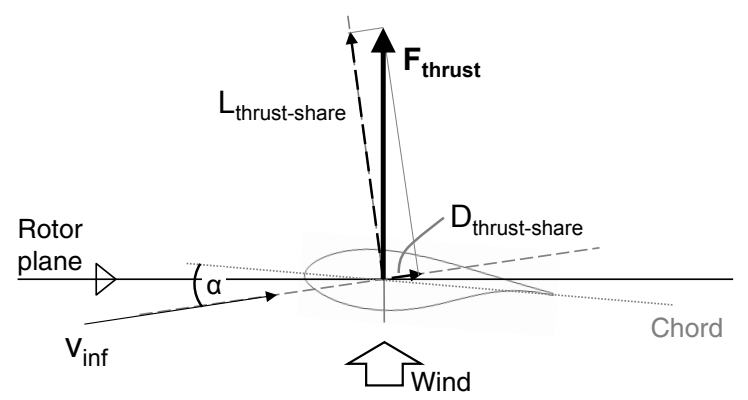

(b)

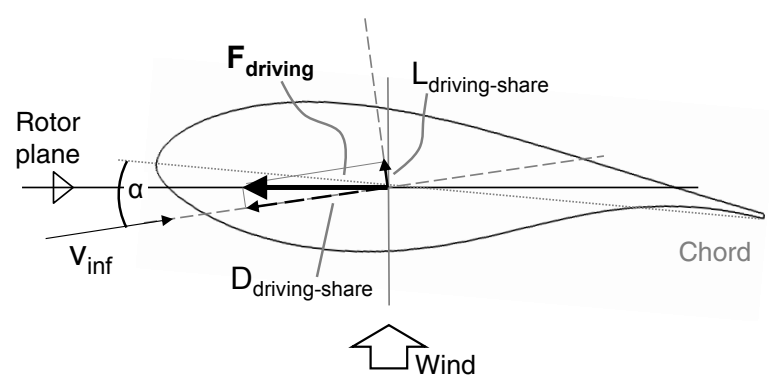

Figure 19. Transformation of driving force and thrust to local aerodynamic coordinate system (driving force, $\mathbf{a}$; thrust, $\mathbf{b}$ ).

to use $c_{1, \text { mean }}$ for the comparison to 2-D simulations and the evaluation of the impact of trailing vortices. With respect to $c_{\mathrm{d}}$ it is difficult to clearly distinguish between the part caused by the AoA oscillation and the induced drag from trailed vorticity. In order to judge the impact of trailing vortices on $c_{\mathrm{d}}$, this differentiation is, however, necessary and the part caused by the AoA oscillation needs to be eliminated. The emphasis of the comparison to 2-D simulations is hence on lift and moment coefficient.

In order to quantify the limitations of the average approach with respect to the phase shift, a fast Fourier transformation (FFT) analysis of the results has been performed for the $1 \mathrm{p}$ frequency and the main peak in frequency was analyzed. The averaged and instantaneous solution differ by only $1 \%$ in lift amplitude and $0.26^{\circ}$ in phase shift, which is below the time step resolution of $1.5^{\circ}$. Due to the limitations caused by the time step, size hysteresis effects are only qualitatively judged by comparing the curves; lift amplitudes, however, can be opposed quantitatively.

\subsection{Comparison to 2-D simulations}

To study the unsteady phenomena in more detail and to analyze the main effects in the 3-D case, the instantaneous $c_{1 \text {, mean }}$ and $c_{\mathrm{m}}$ results of the 3-D extraction are compared to 2-D airfoil simulations of the mid-flap position at mean inflow conditions. The mean inflow conditions used as input for all flap frequencies in 2-D are again $\mathrm{AoA}=6.5^{\circ}$ and
Table 4. $c_{1}$ amplitude, $75 \%$ blade cut, 2-D and 3-D results.

\begin{tabular}{lrrrr}
\hline & $1 \mathrm{p}$ & $2 \mathrm{p}$ & $3 \mathrm{p}$ & $6 \mathrm{p}$ \\
\hline 2-D & 0.42 & 0.40 & 0.38 & 0.33 \\
3-D & 0.30 & 0.29 & 0.25 & 0.24 \\
\hline$\Delta c_{1,3-\mathrm{D}} / \Delta c_{1,2-\mathrm{D}}$ & $71 \%$ & $73 \%$ & $66 \%$ & $73 \%$ \\
\hline 2-D, Theodorsen & 0.42 & 0.40 & 0.38 & 0.35 \\
\hline
\end{tabular}

$v_{\text {inf }}=68 \mathrm{~m} \mathrm{~s}^{-1}$. This leads to a Mach number of 0.2 and a Reynolds number of 15.4 million.

Results of the $c_{1}$ comparison between 2-D static deflection, 2-D sinusoidal deflection and 3-D sinusoidal deflection are shown in Fig. 20. In order to get an impression of the influence of AoA oscillation in the $1 \mathrm{p}$ case, additionally an AoA-corrected version of the 2-D sinusoidal oscillation case is plotted which is computed by Eq. (5).

$$
\begin{aligned}
c_{1,2-\mathrm{D} s i n u s, A o A c o r r}(t) & =c_{1,2-\mathrm{D} \operatorname{sinus}}(t) \\
& +2 \pi\left(\alpha_{3 \text {-Dsinus }}(t)-\alpha_{\text {mean }}\right)
\end{aligned}
$$

As for the higher frequencies the reduced axial velocity method is not applicable, and as the AoA variations are smaller compared to the $1 \mathrm{p}$ case, no AoA-corrected curve is plotted. For these cases the results obtained with the 2-D theory of Theodorsen have been added for comparison. The plots show the expected decrease in lift amplitude from 2D static over 2-D sinusoidal to 3-D sinusoidal. For the $1 \mathrm{p}$ oscillation the comparison of 2-D static and 2-D sinusoidal results shows the minor influence of unsteady effects. Even though hysteresis begins to develop in the 2-D sinusoidal results, the $c_{1}$ amplitude reduction is still small. This result corresponds well to the low reduced frequency in this case of 0.024. Larger differences are seen by comparing 2-D and 3D results which show the decrease in amplitude caused by trailing and shed vorticity. The reduced amplitude leads to less shed vorticity and thus less hysteresis is apparent compared to the 2-D solution.

The AoA-corrected 2-D curve shows the approximate result for a 2-D simulation including an AoA variation in the inflow. The curve demonstrates less hysteresis and a smaller amplitude compared to the baseline progression, which is reasonable since the AoA progression is a feedback of the aerodynamic forcing in the 3-D case. Like it was noticed in Sect. 4.2, for low flap frequencies the axial induction is able to react to the instantaneous load and mimics the effects. The smaller slope which is seen in the 3-D curve can be explained by the decrease in the gradient $d c_{1} / d \beta$ caused by trailing vortices in 3-D.

In 2-D unsteady effects constantly increase with the flap frequency for the regarded cases, which corresponds well to the results with Theodorsen's 2-D theory. The ampli- 
(a)

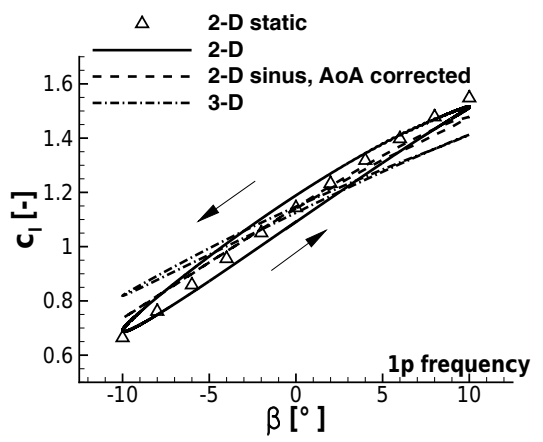

(c)

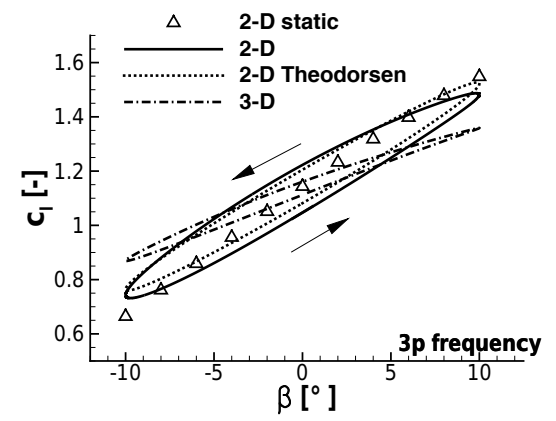

(b)

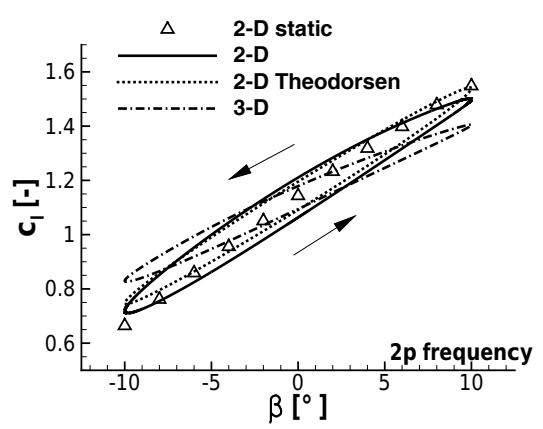

(d)

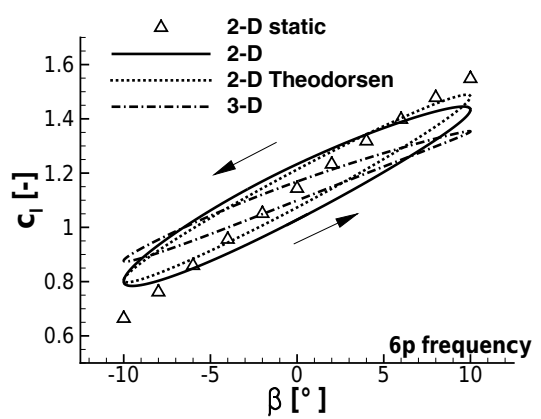

Figure 20. Comparison of 2-D/3-D lift for different flap frequencies: $1 p(\mathbf{a}), 2 p(\mathbf{b}), 3 p(\mathbf{c})$ and $6 p(\mathbf{d})$.

Table 5. $c_{1}$ phase shift, $75 \%$ blade cut, 2-D results.

\begin{tabular}{lrrrr}
\hline & $1 \mathrm{p}$ & $2 \mathrm{p}$ & $3 \mathrm{p}$ & $6 \mathrm{p}$ \\
\hline 2-D & -6.3 & -10.2 & -12.9 & -17.7 \\
2-D, Theodorsen & -4.9 & -7.5 & -9.2 & -11.7 \\
\hline
\end{tabular}

tude of lift oscillation is continuously reducing and more pronounced hysteresis is seen. The results obtained by the Theodorsen's theory are in fair agreement with the CFD results. A more symmetrical hysteresis is apparent compared to the slightly bent CFD curves. Generally, the hysteresis direction is in agreement with the observations made by Troldborg (2005). In 3-D, the amplitude is also continuously decreasing but hysteresis effects show no clear trend. A slightly bigger hysteresis is seen for the $2 p$ frequency than for the $3 p$ frequency. A reason for this phenomenon could be a different phase lag in the AoA oscillation resulting from the flap deflection. For clarification, an AoA extraction for unsteady cases would be required.

Table 4 lists amplitudes and Table 5 phase lags of the lift coefficient for the different cases in order to quantify the effects. The values were again obtained by applying an FFT on the unsteady lift progression and analyzing the main peak in the results. Additionally, results of the 2-D theory by Theodorsen described in Sect. 2.3 are shown in the table. The results of this simplified theory are in very good agreement with the results of 2-D simulations with regard to the $c_{1}$ amplitude. Only at $6 \mathrm{p}$ is a slight difference noticeable. Larger differences are observed in the phase shift, for which higher values are determined in the 2-D CFD simulation. These differences can be caused by the assumption of thin airfoils in Theodorsen's theory as similar observations were found by Motta et al. (2015) for pitching airfoils.

The comparison of the 2-D and 3-D lift amplitudes shows the expected reduction due to the influence of trailing vortices. The 3-D lift amplitude alleviates $66-73 \%$ of the result of 2-D simulations at the same flap frequency based on the round values listed in Table 4 . As it was noticed before in Sect. 4.1 with regard to the thrust oscillation, the lift amplitude of the 3 and $6 \mathrm{p}$ case is similar. This is also seen in the relative amplitude reduction, as $3 p$ shows a significantly lower value than the other cases. Unfortunately, the reason for this phenomenon could not finally be clarified since this would require a method to extract the AoA in transient cases, too. This would allow for judging and comparing the AoA oscillation between all 3-D cases, which provides further insight. Further research is required in this respect. Nevertheless, this relative reduction is roughly constant throughout all flap frequencies. An earlier investigation (Jost et al., 2016) focused on the impact of steady flap deflections on the blade performance. Various flap extensions along the blade radius (10 and $20 \%$ ) and chord (10 and $30 \%$ ) were analyzed in a parametric study at $15 \mathrm{~m} \mathrm{~s}^{-1}$ wind speed. The analysis also included a comparison to 2-D simulations and an extract of the results is shown in Table 6 for positive and negative deflections. A correlation to the results of the present study can be noticed. The relative amplitude reduction $\left(\Delta c_{1,3-\mathrm{D}, 10} \% \mathrm{span} / \Delta c_{1,2-\mathrm{D}}\right)$ is very similar for both 
Table 6. $c_{1}$ amplitude, $75 \%$ blade cut, $15 \mathrm{~m} \mathrm{~s}^{-1}$, steady deflections (Jost et al., 2016).

\begin{tabular}{lrr|rr}
\hline & \multicolumn{2}{c|}{$\beta=10^{\circ}$} & \multicolumn{2}{c}{$\beta=-10^{\circ}$} \\
\cline { 2 - 5 } & $10 \%$ chord & $30 \%$ chord & $10 \%$ chord & $30 \%$ chord \\
\hline$\Delta c_{1,3-\mathrm{D}, 10 \% \operatorname{span} / \Delta c_{1,2-\mathrm{D}}}$ & $70 \%$ & $69 \%$ & $65 \%$ & $65 \%$ \\
$\Delta c_{1,3-\mathrm{D}, 20 \% \operatorname{span} / \Delta c_{1,2-\mathrm{D}}}$ & $80 \%$ & $79 \%$ & $75 \%$ & $77 \%$ \\
\hline
\end{tabular}

(a)

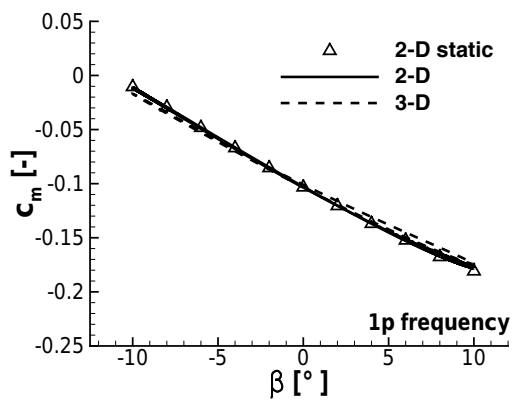

(b)

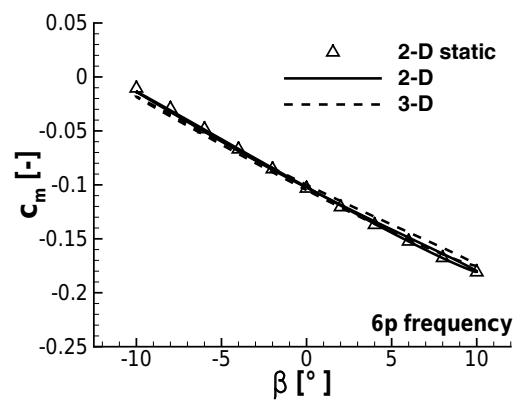

Figure 21. Comparison of 2-D/3-D moment coefficient for different flap frequencies: $1 \mathrm{p}$ (a) and $6 \mathrm{p} \mathrm{(b).}$

chord extents and in a comparable range as observed in the oscillating cases. Please note that the slightly lower values for steady deflections can be explained by the adjusting axial induction as described in Sect. 4.2. The value for $20 \%$

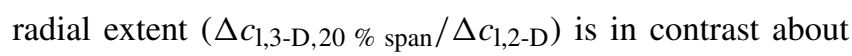
$10 \%$ higher. Consequently, the relative lift amplitude reduction at mid-flap position $\left(\Delta c_{1,3-\mathrm{D}} / \Delta c_{1,2-\mathrm{D}}\right)_{\text {mid, flap }}$ can serve as a rough characteristic value for a certain flap layout. It also allows a decoupled consideration of 3-D and unsteady effects at this location. This means that as a first estimation $\left(\Delta c_{1,3-\mathrm{D}} / \Delta c_{1,2-\mathrm{D}}\right)_{\text {mid,flap }}$ can be determined based on a 3-D simulation with static maximum flap deflection. The amplitude reduction by shed vorticity can be investigated separately in 2-D and can then be superimposed with the 3-D result. This simplified or quasi-2-D approach with regard to shed vorticity is, however, only valid at the mid-flap area. Closer to the flaps edges the unsteady effects will also become three-dimensional.

With respect to a more general aerodynamic modeling in lower fidelity tools, the present investigation suggests a simplified consideration of near-wake effects. The dominant phenomena could be assigned to trailing and shed vorticity, which can be captured by such approaches. First comparisons of the present simulations to the near-wake model by Pirrung et al. (2016), an implementation in the BEM code HAWC2 by DTU (Technical University of Denmark), can be found in Barlas et al. (2016). Aparicio et al. (2016a) have also published favorable results following a similar approach in the BEM code FAST, which was developed by NREL (National Renewable Energy Laboratory). Further benchmarks were performed within the AVATAR project (Manolesos and Prospathopoulus, 2015; Ferreira et al., 2015; Aparicio et al., 2016b).

The results of the moment coefficient $c_{\mathrm{m}}$ are depicted in Fig. 21 for the 1 and $6 \mathrm{p}$ frequency. It is noted that while the previous plots of the 3-D torsion moment were evaluated relative to the pitch axis, the results have been transferred to the quarter chord point for the 2-D comparison. In general, only a minor influence of unsteady aerodynamic effects is observed. A slight reduction of the amplitude and small hysteresis is seen in the 3-D case. The 2-D results are in good agreement with Ferreira et al. (2015), for which similar simulations have been performed for the same airfoil.

\section{Conclusions}

In the present work the influence of unsteady 3-D effects on trailing edge flaps has been investigated for the case of harmonic oscillations of the flap angle. For this purpose four different flap frequencies ranging from 1 to $6 \mathrm{p}$ of the rotational frequency were simulated on a 2-D airfoil and 3-D rotor. The simulations of the 3-D rotor showed a significant influence of trailing and shed vorticity. This leads to a reduction of the amplitude and a phase lag in the load response to the flap signal with increasing flap frequency. This behavior was observed in both integral and sectionally distributed forces along the blade radius. The negative influence of trailing vortices in the flap section is, however, partly compensated for by an increased efficiency of neighboring sections due to the induced up- or downwash. A high impact of dynamic inflow effects is observed in the comparison to steady flap deflections. Unlike for the oscillating cases, the axial induction of the turbine is able to fully adjust to the changed load situation for steady flap deflections, which is noticeable in the rotor loads. 
A detailed investigation of the $75 \%$ blade cut representing the mid-flap position was performed in order to compare 2D airfoil to 3-D rotor results and thereby quantify unsteady 2-D and 3-D effects. The 2-D CFD simulations were additionally compared to the simplified model by Theodorsen, a widespread analytical approach for these matters. A good agreement between 2-D CFD and the Theodorsen model was observed with regard to the amplitude of the lift oscillations. Higher discrepancies could be noticed in the phase shift which could be assigned to Theodorsen's assumption of thin airfoils. The comparison of the 2-D and 3-D CFD simulations revealed the significantly higher reduction of the lift amplitude in the 3-D rotor case due to trailing vorticity. Only about $70 \%$ of the 2-D value for the respective flap frequency is achieved. Additionally, less hysteresis is seen at the rotor blade, which is connected to the decrease in lift amplitude. Two-dimensional and three-dimensional results regarding the moment coefficient were also compared and exhibited an only minor influence of unsteady effects.

As discussed in the present study, an overall CFD-based assessment of the flap concept requires the incorporation of aero-elasticity. On a flexible blade the flap oscillation will be superimposed with pitching and plunging motions. A careful evaluation of these phenomena in comparison to the present investigation will allow an isolation of the different effects and lead to a deeper insight into the 3-D characteristics of trailing edge flaps on a real wind turbine rotor. This is the next step in the ongoing research.

Data availability. The surface description of the DTU $10 \mathrm{MW}$ turbine is available online (http://dtu-10mw-rwt.vindenergi.dtu.dk). Simulation results can be provided by contacting the corresponding author or Thorsten Lutz (lutz@iag.uni-stuttgart.de).

Competing interests. The authors declare that they have no conflict of interest.

Acknowledgements. The authors acknowledge European FP7 project INNWIND.EU for funding (grant agreement no. 308974). This work was partly performed on the Supermuc Cluster (LRZ Munich), the HazelHen and NEC Cluster (HLRS Stuttgart) and the bwUniCluster (framework bwHPC; funding: Ministry of Science, Research and Arts and the Universities of the state of Baden-Württemberg).

Edited by: C. L. Bottasso

Reviewed by: V. A. Riziotis and one anonymous referee

\section{References}

Aparicio, M., González, A., Gomez-Iradi, S., and Munduate, X.: Development of an engineering code for the implementation of aerodynamic control devices in BEM, Journal of Physics: Conference Series, 753, 082001, available at: http://stacks.iop. org/1742-6596/753/i=8/a=082001 (last access: 10 May 2017), $2016 a$.

Aparicio, M., Martín, R., Muñoz, A., and González, A.: AVATAR D3.3 - Results of a parametric study of flow devices, guidelines for design, Tech. rep., AVATAR project, available at: http://www.eera-avatar.eu/fileadmin/avatar/user/V28D3. 3AVATAR_D33.pdf (last access: 10 May 2017), 2016 b.

Bak, C., Zahle, F., Bitsche, R., Kim, T., Yde, A., Henriksen, L., Andersen, P., Natarajan, A., and Hansen, M.: Design and performance of a $10 \mathrm{MW}$ turbine, Tech. rep., DTU, available at: http: //dtu-10mw-rwt.vindenergi.dtu.dk (last access: 10 May 2017), 2013.

Bangga, G., Kim, Y., Lutz, T., Weihing, P., and Krämer, E. E.: Investigations of the inflow turbulence effect on rotational augmentation by means of CFD, Journal of Physics: Conference Series, 753, 022026, available at: http://stacks.iop.org/1742-6596/753/ $\mathrm{i}=2 / \mathrm{a}=022026$ (last access: 10 May 2017), 2016.

Barlas, T., van der Veen, G., and van Kuik, G.: Model predictive control for wind turbines with distributed active flaps: incorporating inflow signal and actuator constraints, Wind Energy, 15, 757-771, doi:10.1002/we.503, 2012a.

Barlas, T., Zahle, F., Sørensen, N., Gaunaa, M., and Bergami, L.: Simulations of a rotor with active deformable trailing edge flaps in half-wake inflow: Comparison of EllipSys 3D with HAWC2, in: European Wind Energy Conference (EWEC), 2012b.

Barlas, T., Jost, E., Pirrung, G., Tsiantas, T., Riziotis, V., Navalkar, S., Lutz, T., and van Wingerden, J.-W.: Benchmarking aerodynamic prediction of unsteady rotor aerodynamics of active flaps on wind turbine blades using ranging fidelity tools, Journal of Physics: Conference Series, 753, 022027, available at: http://stacks.iop.org/1742-6596/753/i=2/a=022027 (last access: 10 May 2017), 2016.

Bergami, L. and Gaunaa, M.: ATEFlap Aerodynamic Model, a dynamic stall model including the effects of trailing edge flap deflection, Tech. rep., DTU - Technical University of Denmark, available at: http://orbit.dtu.dk/files/6599679/ris-r-1792.pdf (last access: 10 May 2017), 2012.

Bergami, L., Riziotis, V., and Gaunaa, M.: Aerodynamic response of an airfoil section undergoing pitch motion and trailing edge flap deflection: a comparison of simulation methods, Wind Energy, 18, 1273-1290, doi:10.1002/we.1759, 2015.

Bernhammer, L., von Kuik, G., and Breuker, R. D.: Fatigue and extreme load reduction of wind turbine components using smart rotors, J. Wind Eng. Ind. Aerod., 154, 84-95, doi:10.1016/j.jweia.2016.04.001, 2016.

Castaignet, D., Barlas, T., Buhl, T., Poulsen, N., Wedel-Heinen, J., Olsen, N., Bak, C., and Kim, T.: Full-scale test of trailing edge flaps on a Vestas V27 wind turbine: active load reduction and system indentification, Wind Energy, 17, 549-564, doi:10.1002/we.1589, 2014.

Chen, Z., Stol, K., and Mace, B.: Wind turbine blade optimisation with individual pitch and trailing edge flap control, Renew. Energ., 103, 750-765, doi:10.1016/j.renene.2016.11.009, 2017. 
Daynes, S. and Weaver, P.: A morphing trailing edge device for a wind turbine, J. Intel. Mat. Syst. Str., 23, 691-701, doi:10.1177/1045389X12438622, 2012.

Ferreira, C., Gonzalez, A., Baldacchino, D., and Aparicio, M.: AVATAR D3.2 - Development of aerodynamic codes for modelling of flow devices on aerofoils and rotors, Tech. rep., AVATAR project, available at: http://www.eera-avatar. eu/fileadmin/avatar/user/Report_task_3p2_version_4_.pdf (last access: 10 May 2017), 2015.

Hariharan, N. and Leishman, J.: Unsteady aerodynamics of a flapped airfoil in subsonic flow by indicial concepts, J. Aircraft, 33, 855-868, doi:10.2514/3.47028, 1996.

Johansen, J. and Sørensen, N.: Aerofoil characteristics from 3D rotor CFD simulations, Wind Energy, 7, 283-294, doi:10.1002/we.127, 2004.

Jost, E., Barlas, T., Riziotis, V., and Navalkar, S.: Innwind D2.3.2 - Validation of New Control Concepts by Advanced FluidStructure Interaction Tools, Tech. rep., Innwind, EU project, 2015a.

Jost, E., Lutz, T., and Krämer, E.: Steady and unsteady CFD power curve simulations of generic $10 \mathrm{MW}$ turbines, in: Proceedings of the 11th PhD Seminar on Wind Energy in Europe, available at: http://www.eera-avatar.eu/fileadmin/avatar/ user/ExtendedAbstract_EvaJost.pdf (last access: 10 May 2017), $2015 b$.

Jost, E., Lutz, T., and Krämer, E.: A parametric CFD study of morphing trailing edge flaps applied on a $10 \mathrm{MW}$ offshore wind turbine, Energy Procedia, 94, 53-60, doi:10.1016/j.egypro.2016.09.192, 2016.

Jost, E., Beckers, M., Lutz, T., and Krämer, E.: CFD study of trailing edge flaps for load control on wind turbines, New Results in Numerical and Experimental Fluid Mechanics - Contributions to the STAB symposium Braunschweig, under review, 2017.

Klein, L., Lutz, T., and Krämer, E.: CFD analysis of 2-bladed wind turbine, in: Proceedings of the 10th PhD Seminar on Wind Energy in Europe, available at: https://eawephdseminar. sciencesconf.org/conference/eawephdseminar/pages/eda_en.pdf (last access: 10 May 2017), 2014.

Kroll, N. and Fassbender, J.: MEGAFLOW - Numerical Flow Simulation for Aircraft Design, Springer, 2002.

Leble, V., Wang, Y., and Barakos, G.: CFD analysis of 10-MW turbines, in: German Wind Energy Conference (DEWEK), 2015.

Leishman, J.: Principles of Helicopter Aerodynamics, Cambridge University Press, Cambridge, UK, 2006.

Leishman, J. G.: Unsteady Lift of a Flapped Airfoil by Indicial Concepts, J. Aircraft, 31, 288, doi:10.2514/3.46486, 1994.

Leishman, J. G.: Challenges in Modelling the Unsteady Aerodynamics of Wind Turbines, Wind Energy, 5, 85-132, doi:10.1002/we.62, 2002.

Madsen, H., Andersen, P., Andersen, T., Bak, C., and Buhl, T.: The potentials of the controllable rubber trailing edge flap (CRTEF), in: Proceedings of the EWEC, available at: http: //orbit.dtu.dk/files/4556206/Madsen_paper_ewec_2010.pdf (last access: 10 May 2017), 2010.
Manolesos, M. and Prospathopoulus, J.: AVATAR D3.1 - CFD and experimental database of flow devices, comparison, Tech. rep., AVATAR project, 2015.

Menter, F. R.: Two-Equation Eddy-Viscosity Turbulence Models for Engineering Applications, AIAA Journal, 32, 1598-1605, 1994.

Motta, V., Guardone, A., and Quaranta, G.: Influence of airfoil thickness on unsteady aerodynamic loads on pitching airfoils, J. Fluid Mech., 774, 460-487, 2015.

Pirrung, G., Madsen, H., Kim, T., and Heinz, J.: A coupled near and far wake model for wind turbine aerodynamics, Wind Energy, 19, 2053-2069, doi:10.1002/we.1969, 2016.

Schuff, M., Kranzinger, P., Keßler, M., and Krämer, E.: Advanced CFD-CSD coupling: Generalized, high performant, radial basis function based volume mesh deformation algorithm for structured, unstructured and overlapping meshes, in: 40th European Rotorcraft Forum, Southhampton, 2014.

Schulz, C., Letzgus, P., Lutz, T., and Krämer, E.: CFD study of yawed inflow on loads, power and near wake of a generic wind turbine, Wind Energy, 20, 253-268, doi:10.1002/we.2004, 2016.

Sørensen, N., Hansen, N., Garcia, N., Florentie, L., Boorsma, K., Gomez-Iradi, S., Prospathopoulus, J., Barakos, G., Wang, Y., Jost, E., and Lutz, T.: AVATAR D2.3 - Power curve predictions, Tech. rep., AVATAR project, available at: http://www. eera-avatar.eu/fileadmin/mexnext/user/report-d2p3.pdf (last access: 10 May 2017), 2015.

Theodorsen, T.: General Theory of Aerodynamic Instability and the Mechanism of Flutter, Tech. Rep. 496, NACA, available at: https: //ntrs.nasa.gov/archive/nasa/casi.ntrs.nasa.gov/19930090935.pdf (last access: 10 May 2017), 1935.

Theodorsen, T. and Garrick, I. E.: Nonstationary flow about a wingaileron-tap combination including aerodynamic balance, Tech. rep., NACA, available at: https://ntrs.nasa.gov/archive/nasa/casi. ntrs.nasa.gov/19930091815.pdf (last access: 10 May 2017), 1942.

Troldborg, N.: Computational study of the Ris $\varnothing-\mathrm{B} 1-18$ airfoil with a hinged flap providing variable trailing edge geometry, Wind Engineering, 2, 89-113, 2005.

Ungurán, R. and Kühn, M.: Combined individual pitch and trailing edge flap control for structural load alleviation of wind turbines, in: 2016 American Control Conference, doi:10.1109/ACC.2016.7525262, 2016.

Wolff, T., Ernst, B., and Seume, J.: Aerodynamic behavior of an airfoil with morphing trailing edge for wind turbine applications, Journal of Physics: Conference Series, 524, 012018, available at: http://iopscience.iop.org/article/10.1088/ 1742-6596/524/1/012018/pdf (last access: 10 May 2017), 2014. 